\title{
SOME ENERGY INEQUALITIES FOR TWO-BODY PROBLEMS AND HELIUM DIMER
}

\author{
S. KILIĆ AND B. KRIVIĆ \\ Faculty of Natural Science, University of Split, 21000 Split, Croatia \\ (Received October 10, 1997; revised version December 8, 1997; \\ in final form January 12, 1998)
}

\begin{abstract}
It is shown that the energy of two particles in 2D (2 dimensions) is always less or equal than energy in $1 \mathrm{D}$, and the energy in $3 \mathrm{D}$ can be less or greater than in 2D. The application to the important case of helium 4 dimer shows that this molecule may have only in $2 \mathrm{D}$ at least one bound excited state
\end{abstract}

PACS numbers: $36.90 .+\mathbf{f}$

\section{Introduction}

In many microscopic theories of matter it is important to know the solution of the two-body problem. This is the case in statistical mechanics, scattering theory, quantum fluids and others. For example, the microscopic theories of quantum fluids include some approximations related to the study of the two-body problem. Namely, the many-body wave function in the Jastrow approximation is a product of two-body functions for each pair of particles (or generally in the Feenberg form it is a symmetrized product of any order of the correlation among particles [1-7]). It is known that short range correlations have been described in this well. A quite new example is the helium 4 dimer, which was experimentally discovered four years ago [8] and recently approved [9, 10]. Much theoretical and experimental research concerning this two-body system should be done in future.

Therefore it is very useful to have some general relations regarding the two-body problem. Particularly, if the problem can be solved in lower dimensions it would be useful to draw conclusions for higher dimensions.

In Sec. 2 we perform several useful relations which have been applied to the helium 4 dimer in Sec. 3. 


\section{Inequalities between energies in different dimensions}

Consider two identical particles which interact via a spherically symmetrical potential $\widehat{V}\left(r_{1}, r_{2}\right)$. In the relative coordinates the Hamiltonian of the system reads

$$
\widehat{H}=-\frac{\hbar^{2}}{m} \Delta+\widehat{V}(r)
$$

where $m$ is the mass of a particle and the operator $\Delta$ has the form

$$
\begin{aligned}
& \Delta_{1}=\frac{\mathrm{d}^{2}}{\mathrm{~d} r^{2}} \text { in } 1 \mathrm{D}, \\
& \Delta_{2}=\frac{\partial^{2}}{\partial r^{2}}+\frac{1}{r} \frac{\partial}{\partial r}+\frac{1}{r^{2}} \frac{\partial^{2}}{\partial \varphi^{2}} \quad \text { in } 2 \mathrm{D}, \\
& \Delta_{3}=\frac{\partial^{2}}{\partial r^{2}}+\frac{2}{r} \frac{\partial}{\partial r}+\frac{1}{r^{2}} \frac{\partial^{2}}{\partial \vartheta^{2}}+\frac{1}{r^{2}} \cot \vartheta \frac{\partial}{\partial \vartheta}+\frac{1}{r^{2} \sin ^{2} \vartheta} \frac{\partial^{2}}{\partial \varphi^{2}} \quad \text { in 3D. }
\end{aligned}
$$

In order to obtain relations between energies in different dimensions we employ variational ansatz. For all cases it may be written in the form

$$
E_{n} \leq \frac{\int \Psi_{n}^{*} \widehat{H}_{n} \Psi_{n} r^{n-1} \mathrm{~d} r \mathrm{~d} \Omega_{n}}{\int \Psi_{n}^{*} \Psi_{n} r^{n-1} \mathrm{~d} r \mathbb{N} \Omega_{n}}
$$

where $n=1,2,3$ denotes the dimension of physical space, $\Psi_{n}$ and $\widehat{H}_{n}$ are corresponding trial functions and the Hamiltonians, $\mathrm{d} \Omega_{1}=1, \mathrm{~d} \Omega_{2}=\mathrm{d} \varphi$, and $\mathrm{d} \Omega_{3}=$ $\sin \vartheta \mathrm{d} \vartheta \mathrm{d} \varphi$. From the relations (1-4) it follows

$$
\begin{aligned}
& \widehat{H}_{1}=-\frac{\hbar^{2}}{m} \frac{\mathrm{d}^{2}}{\mathrm{~d} r^{2}}+\widehat{V} \\
& \widehat{H}_{2}=\widehat{H}_{1}-\frac{\hbar^{2}}{m}\left(\frac{1}{r} \frac{\partial}{\partial r}+\frac{1}{r^{2}} \frac{\partial^{2}}{\partial \varphi^{2}}\right), \\
& \widehat{H}_{3}=\widehat{H}_{2}-\frac{\hbar^{2}}{m}\left(\frac{1}{r} \frac{\partial}{\partial r}+\frac{1}{r^{2}} \frac{\partial^{2}}{\partial \vartheta^{2}}+\frac{1}{r^{2}} \cot \vartheta \frac{\partial}{\partial \vartheta}+\frac{1}{r^{2}} \cot \vartheta^{2} \frac{\partial^{2}}{\partial \varphi^{2}}\right) .
\end{aligned}
$$

Suppose that radial and angular motions can be separated both in 2D and 3D. The wave functions can then be factorized as follows:

$$
\begin{aligned}
& \Psi_{2}=\Psi_{2}(r) \Psi_{2}(\varphi), \\
& \Psi_{3}=\Psi_{3}(r) \Psi_{3}(\varphi) \Psi_{3}(\vartheta) .
\end{aligned}
$$

The wave functions $\Psi_{3}(\varphi) \approx \Psi_{2}(\varphi)$ and $\Psi_{3}(\vartheta)$ are solutions of eigenvalue problems

$$
\begin{aligned}
& \frac{\partial^{2} \Psi_{2}(\varphi)}{\partial \varphi^{2}}=-\mu^{2} \Psi_{2}(\varphi), \\
& \left(\frac{\partial^{2}}{\partial \vartheta^{2}}+\cot \vartheta \frac{\partial}{\partial \vartheta}-\mu^{2} \cot \vartheta^{2}\right) \Psi_{3}(\vartheta)=\lambda \Psi_{3}(\vartheta) .
\end{aligned}
$$

Solving above relations, one finds $\lambda=\mu^{2}-l(l+1), \mu=0,1,2, \ldots l, l=0,1,2, \ldots$ Since $\Psi_{1}(r) \approx \Psi_{2}(r) \approx \Psi_{3}(r)$ it follows

$$
E_{2} \leq \frac{\int_{0}^{\infty} \mathrm{d} r r \Psi_{1}^{*}(r) \widehat{H}_{1} \Psi_{1}(r)}{\int_{0}^{\infty} \mathrm{d} r r \Psi_{1}^{*}(r) \Psi_{1}(r)}-\frac{\hbar^{2}}{m} \frac{\int_{0}^{\infty} \mathrm{d} r \Psi_{1}^{*}(r) \partial \Psi_{1}(r) / \partial r}{\int_{0}^{\infty} \mathrm{d} r \Psi_{1}^{*}(r) \Psi_{1}(r)}
$$




$$
\begin{aligned}
& +\frac{\hbar^{2}}{m} \mu^{2} \frac{\int_{0}^{\infty} \mathrm{d} r \Psi_{1}^{*}(r) \Psi_{1}(r) / r}{\int_{0}^{\infty} \mathrm{d} r r \Psi_{1}^{*}(r) \Psi_{1}(r)} \\
E_{3} \leq & \frac{\int_{0}^{\infty} \mathrm{d} r r^{2} \int_{0}^{2 \pi} \mathrm{d} \varphi \Psi_{2}^{*}(r) \Psi_{2}^{*}(\varphi) \widehat{H}_{2} \Psi_{2}(r) \Psi_{2}(\varphi)}{\int_{0}^{\infty} \mathrm{d} r r^{2} \int_{0}^{2 \pi} \mathrm{d} \varphi \Psi_{2}^{*}(r) \Psi_{2}^{*}(\varphi) \Psi_{2}(r) \Psi_{2}(\varphi)}-\frac{\hbar^{2}}{m} \frac{\int_{0}^{\infty} \mathrm{d} r r \Psi_{2}^{*}(r) \partial \Psi_{2}(r) / \partial r}{\int_{0}^{\infty} \mathrm{d} r r^{2} \Psi_{2}^{*}(r) \Psi_{2}(r)} \\
& +\frac{\hbar^{2}}{m}\left[l(l+1)-\mu^{2}\right] \frac{\int_{0}^{\infty} \mathrm{d} r \Psi_{2}^{*}(r) \Psi_{2}(r)}{\int_{0}^{\infty} \mathrm{d} r r^{2} \Psi_{2}^{*}(r) \Psi_{2}(r)}
\end{aligned}
$$

The relations (13) and (14) are quite general. In a particular case when $\Psi_{1}$ and $\Psi_{2}$ are eigenfunctions of Hamiltonians $\widehat{H}_{1}$ and $\widehat{H}_{2}$, the first term on the right hand side in (13) and (14) becomes equal to the eigenvalues $\varepsilon_{1}$ and $\varepsilon_{2}$, respectively. If, in addition, the wave functions are real and angular motion is absent, then

$$
\begin{aligned}
& E_{2} \leq \varepsilon_{1}, \\
& E_{3} \leq \varepsilon_{2}+\frac{\hbar^{2}}{2 m} \frac{\int_{0}^{\infty} \mathrm{d} r \Psi_{2}^{2}(r)}{\int_{0}^{\infty} \mathrm{d} r r^{2} \Psi_{2}^{2}(r)} .
\end{aligned}
$$

It is evident that the energy in $2 \mathrm{D}$ is never greater than in $1 \mathrm{D}$. While the energy in $3 \mathrm{D}$ may be also greater than in $2 \mathrm{D}$.

\section{More about the helium 4 dimer}

As it is known helium 4 dimer is a molecule which was observed experimentally in 1993 [8]. This dimer is the largest one that we know. The average distance of atoms is about $50 \AA$ in the ground state. In our opinion the existence of excited states in 3D is still questionable. The existence of at least one excited state of this dimer in 2D was proved in our paper [11]. This quite novel result and above inequalities are employed here to conclude something about excited states in $3 \mathrm{D}$. To reach this goal and make the presentation clear we consider two cases particularly.

\subsection{Ground state}

We take the variational trial wave function which we used in Refs. $[11,12]$

$$
\Psi_{0}(r)=\exp \left[-(\alpha / r)^{5}\right] \exp (-s r) \text {. }
$$

The first exponential term on the right hand side describes short range correlation between atoms and it was used in the theory of liquid helium, for instance in Refs. [2, 4]; it is known as the Feenberg-Wu-Ljolje wave function. The second factor in the trial wave function (17) takes care on long range correlation and keeps atoms in a finite distance. Using Lennard-Jones and several modern potentials we showed that dimer is bound in 2D. For the Aziz "best" potential HFD-B3-FCI1 $[13,14]$

$$
\begin{aligned}
& V(x)=\epsilon V^{*}(x), \\
& V^{*}(x)=A \exp \left(-\alpha x+\beta x^{2}\right)-\left(\frac{c_{6}}{x^{6}}+\frac{c_{8}}{x^{8}}+\frac{c_{10}}{x^{10}}\right) F(x),
\end{aligned}
$$




$$
F(x)=\left\{\begin{array}{ll}
\exp \left[-(D / x-1)^{2}\right], & x<D \\
1, & x \geq D
\end{array},\right.
$$

where $x=r / r_{m}$ and $\epsilon=15.1265 \times 10^{-23} \mathrm{~J}, D=1.438, r_{m}=2.9683 \times 10^{-10} \mathrm{~m}$, $c_{6}=1.35186623, A=186924.404, c_{8}=0.41495143, \alpha=10.5717543$, $c_{10}=0.17151143, \beta=-2.07758779$, the binding energy in the ground state is obtained to be $0.054311 \times 10^{-23} \mathrm{~J}$ with the minimization parameters $\alpha=2.64 \times 10^{-10} \mathrm{~m}$ and $s=0.098 \times 10^{10} \mathrm{~m}^{-1}$.

Although the binding of two atoms in $3 \mathrm{D}$, for the same class of radial wave functions and zero angular momentum, is not found, we can estimate the quality of our calculation and use it later for an excited state. (Of course another appropriate class of trial wave functions could give a bound state in 3D, but they are unknown). As the dimer was discovered experimentally with a binding energy of $1 \mathrm{mK}[8]$, or calculated $1.7 \mathrm{mK}$ [15], one may estimate the trial function using the relation (14). Namely, on the right hand side of the relation (14) only a part of the first term and the second term survive for $l=0$. The calculated values of these terms are: $-39.337 \mathrm{mK}$ and $+61.2243 \mathrm{mK}$ for the first and the second term, respectively. It means that the absolute value of the first term is about $2 / 3$ of the second term. Since the experimental value of $E_{3}$ is $-1 \mathrm{mK}$, what is the left hand side of the relation (14), one may conclude that the function $\Psi_{2}(r)$, used as a trial function, leads to the result which is about $67 \%$ of the right value. (Evidently the "error" of about $33 \%$ comes out because of the form of our trial function which influences stronger on the kinetic energy term in 3D than in 2D.)

Let us mention that a bound state in 1D has not been found.

\subsection{Excited state}

The existence of a bound excited state has been proved in 2D as well [11]. The trial wave function used is approximately orthogonal to the ground state wave function $\Psi_{0}$, and has the following form:

$$
\Psi_{1}(r)=(1-b r) \Psi_{0}
$$

where the parameter $b$ defines the node. The above form of the excited wave function is known from the theory of the hydrogen atom. From the variational ansatz in a numerical minimization it is obtained: the binding energy $0.00548207 \times$ $10^{-23} \mathrm{~J}, b=0.02273293 \times 10^{10} \mathrm{~m}^{-1}, \alpha=2.61 \times 10^{-10} \mathrm{~m}$ and $s=0.023 \times 10^{10} \mathrm{~m}^{-1}$. For the same parameters the second term on the right hand side of the relation (14) is $0.0074967 \times 10^{-23} \mathrm{~J}$. The lowest value of the third term in the relation (14) is two times greater $(\mu=1$ and $l=1)$ than the second one. It means that the positive term on the right hand side $(+16.2891 \mathrm{mK})$ is at least 4.1 times greater than the negative term $(-3.9705 \mathrm{mK})$. If the quality of the approximation of the trial wave function is the same as in the ground state, this simply means that the bound excited state of the helium dimer in 3D does not exist. This result supports the conclusion in the historical paper [8].

The helium dimer is a fascinating molecule. So light and so big! To demonstrate this in Figs. 1 and 2, 3 the Aziz "best" HFD-B3-FCI1 potential and both trial wave functions are plotted. 

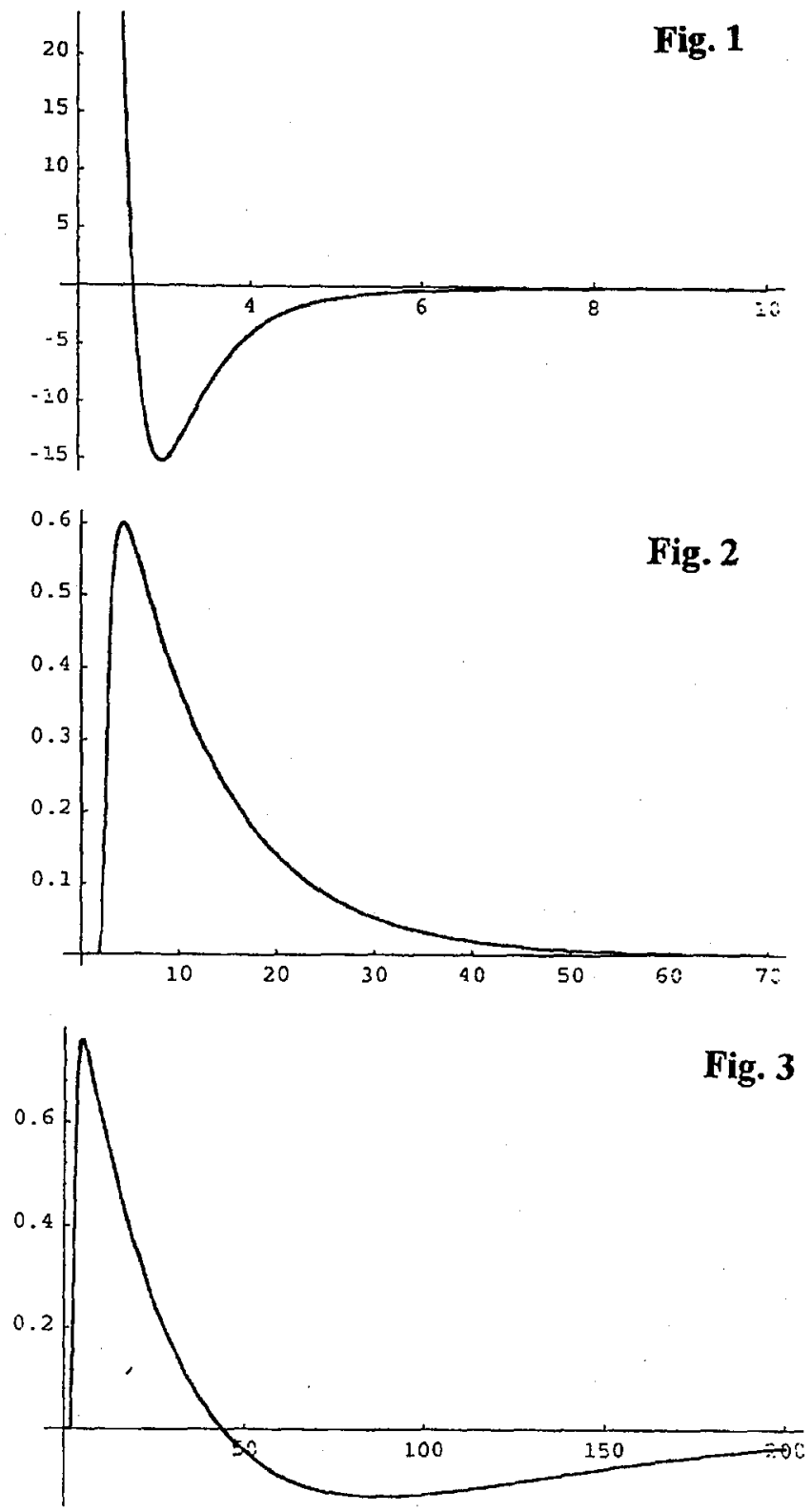

Fig. 1. The Aziz "best" HFD-B3-FCI1 potential between two helium atoms (in $10^{-23} \mathrm{~J}$ ), the distance is plotted in $\AA$.

Fig. 2. The radial ground state wave function, the distance is plotted in $\AA$.

Fig. 3. The radial excited state wave function, the distance is plotted in $\AA$. 
Note that the huge numerical calculation has been done using the Mathematica application.

\section{References}

[1] E.P. Gross, Ann. Phys. (N.Y.) 20, 44 (1962).

[2] K. Ljolje, Fizika 1, 1 (1968).

[3] E. Feenberg, Theory of Quantum Fluids, Academic, New York 1969.

[4] S. Kilić, Fizika 2, 105'(1970).

[5] C.W. Woo, Phys. Rev. Lett. 28, 1442 (1972).

[6] C.E. Campbell, Phys. Lett. A 44, 471 (1973).

[7] E. Feenberg, Ann. Phys. (N.Y.) 84, 128 (1974).

[8] F. Luo, G.C. McBane, G. Kim, C.F. Giese, W.R. Gentry, J. Chem. Phys. 98, 3564 (1993).

[9] F. Luo, C.F. Giese, W.R. Gentry, J. Chem. Phys. 104, 1151 (1996).

[10] W. Schollkopf, J.P. Toennies, J. Chem. Phys. 104, 1155 (1996).

[11] S. Kilić, B. Krivić, submitted for publication.

[12] S. Kilić, S. Sunarić, Fizika 11, 225 (1979).

[13] R.A. Aziz, M.J. Slaman, Metrologia 27, 211 (1990).

[14] A.R. Janzen, R.A. Aziz, J. Chem. Phys. 103, 9626 (1995).

[15] T. Korona, H.L. Williams, R. Bukowski, B. Jeziorski, K. Szalewicz, J. Chem. Phys. 106, 5109 (1997). 